\title{
Modified Function Projective Synchronization of Financial Hyperchaotic Systems via Adaptive Impulsive Controller with Unknown Parameters
}

\author{
Guoliang Cai, Lingling Zhang, Lan Yao, and Xiulei Fang \\ Nonlinear Scientific Research Center, Jiangsu University, Zhenjiang, Jiangsu 212013, China \\ Correspondence should be addressed to Guoliang Cai; glcai@ujs.edu.cn
}

Received 19 December 2014; Accepted 10 March 2015

Academic Editor: Gualberto Solís-Perales

Copyright (C) 2015 Guoliang Cai et al. This is an open access article distributed under the Creative Commons Attribution License, which permits unrestricted use, distribution, and reproduction in any medium, provided the original work is properly cited.

\begin{abstract}
Modified function projective synchronization via adaptive impulsive controller between two different financial hyperchaotic systems is investigated, where the external uncertainties are considered. The updated laws of the unknown parameters are given and the sufficient conditions are deduced based on Lyapunov stability theorem and the stability analysis of impulsive system. Finally, the two financial hyperchaotic systems are taken for example and the numerical examples are worked through for illustrating the main results.
\end{abstract}

\section{Introduction}

Chaos synchronization, an important topic in nonlinear science, has been developed and studied extensively in various fields including secure communication, biological systems, information science, chemical reactions, and plasma technologies [1-3]. Since Pecora and Carroll [4] introduced a method to synchronize two identical systems with different initial conditions, a variety of approaches have been proposed for the synchronization of chaotic systems including complete synchronization [5], phase synchronization [6], generalized synchronization [7], lag synchronization [8], exponential synchronization [9], projective synchronization $[10,11]$, modified function projective synchronization [12], and adaptive synchronization [13].

Among those synchronization methods, in recent years, function projective synchronization (FPS) of special form is introduced by some researches [14], where the drive system and response system could be synchronized up to a scaling function. It was modified in a more general form by $\mathrm{Li}$ and Zhao [15], function projective synchronization, which is called modified function projective synchronization (MFPS), where the drive system and response system are synchronized up to a desire scaling function matrix in the Lorenz system by an active control method. Up to now, MFPS which consider external uncertainties has been proposed by some researchers in [16].

As we all know, financial systems are concerned with our daily life [17]. Chaos will appear when economic crisis happens. Actually, the 2007 global economic crisis shows the existence of the chaos. That is the background of our previous system in this paper [18]. The dynamical behaviors of the system are more complex because they have more than one positive Lyapunov exponent and they are expanded in more than one direction. So an effective and rapid control method is very necessary for government to take when chaotic phenomenon appears. And, in this paper, we study its MFPS via an adaptive impulsive control method in this financial hyperchaotic system.

Impulsive phenomena exist in many biological science and mechanics fields actually. Consequently, the impulsive control becomes an interesting and useful synchronization approach $[19,20]$. Its main idea is to change the states of a system by the "sudden jumps" instantaneously. Impulsive control may provide an efficient method for some cases in which the systems can not endure continuous disturbance. Therefore, by using the impulsive control method, the response system needs to receive the information from the drive system and achieve synchronization faster only at some discrete instants. So it has very strong advantage in practice 
due to reduced control cost and has strong robustness and anti-interference ability. Impulsive control is chosen aiming at applying some simple and easily verified sufficient conditions to stabilize the financial system. It is simpler to achieve practically, and the stabilization time is shorter than adaptive control. To the best of our knowledge, MFPS via impulsive control in this new financial hyperchaotic system has not been studied.

The rest of this paper is organized as follows. In Section 2, the new financial hyperchaotic system is presented. In Section 3, some sufficient conditions for MFPS are derived by impulsive control. In Section 4, MFPS of the financial hyperchaotic systems with uncertain parameters is investigated. Numerical examples are given in Section 5. Finally, the conclusions are drawn in Section 6.

\section{System Description}

In this section, we will give some description of our system studied. The novel nonlinear hyperchaotic finance system [18] can be described by the following differential equations:

$$
\begin{aligned}
& \dot{x}=z+(y-a) x+w, \\
& \dot{y}=1-b y-x^{2}, \\
& \dot{z}=-x-c z, \\
& \dot{w}=-d x y-k w,
\end{aligned}
$$

where the model describes the time variations of four state variables: the interest rate $x$, the investment demand $y$, the price exponent $z$, and the average profit margins $w, a, b, c$, $d$, and $k$ are the parameters of system (1), and they are all positive constants. As parameters are chosen as $a=0.9$, $b=0.2, c=1.5, d=0.2$, and $k=0.17$, the four Lyapunov exponents of system (1) calculated with Wolf algorithm are $L_{1}=0.034432, L_{2}=0.018041, L_{3}=0$, and $L_{4}=-1.1499$, which are presented in Figure 1. Figures 2(a)-2(d) show the 3dimensional phase portraits of financial hyperchaotic system (1) and the time responses of $(x, y, z, w)^{\mathrm{T}}$ in system (1) are presented in Figure 3. System (1) is constructed in the background of the global economic crisis which occurred in 2007 and still exists nowadays. As this global economic crisis did not cause the great depression like in the 1920s1930 s, system (1) is a weak hyperchaotic finance system. The largest Lyapunov exponent of system (1) is a little small, but this hyperchaotic finance system exactly reflects this finance phenomenon. By calculation, hyperchaotic system (1) has three equilibrium points: $P_{0}(0,1 / b, 0,0)$ and $P_{1,2}( \pm \theta,(k+$ $a c k) / c(k-d), \mp \theta / c, d \theta(1+a c) /(c d-c k))$ when the parameters $a, b, c, d$, and $k$ satisfy $(k b+d c+a b c k-c k) / c(d-k)>0$ and $\theta=$ $\sqrt{(k b+a b c k) / c(d-k)+1}$. As the eigenvalues of the Jacobian matrix at the equilibrium point $P_{0}$ are $3.6433,0.1278,-0.200$, and -1.3411, respectively, hence, equilibrium $P_{0}$ is an unstable saddle point in this nonlinear four-dimensional autonomous system. Similarly, system (1) undergoes a Hopf bifurcation at the equilibrium points $P_{1,2}$. Therefore, the equilibrium points $P_{1,2}$ are unstable saddle points. According to the hyperchaotic

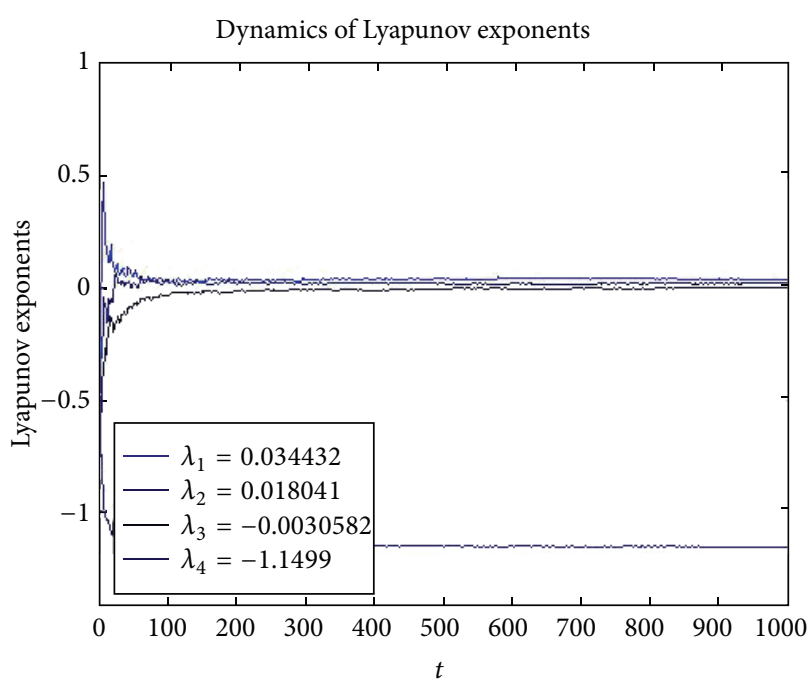

FIGURE 1: Lyapunov exponents spectrum of system (1).

parameter values given above, the equilibrium points are calculated as: $P_{0}(0,5,0,0), P_{1}(1.66,-8.87,-1.11,17.4)$, and $P_{2}(-1.66,-8.87,1.11,-17.4)$. More dynamic behaviors have been analyzed about system (1) in [18].

Remark 1. Sometimes we need to propose some assumptions to simplify mathematical model for complexity of question when we apply this model to discuss some practical questions.

\section{MFPS via Impulsive Control with Parameter Identification}

In this section, we discuss the modified function projective synchronization of the uncertain dynamical system.

Consider a class of chaotic systems with unknown parameters:

$$
\dot{x}=f(x)+\Phi(x) \Theta
$$

where $x \in R^{n}$ denotes the state vector of the system, $\Phi(x): R^{n} \rightarrow R^{n \times p}$, and $\Theta \in R^{p}$ represent the vector of unknown parameters. Take system (2) as the drive system, and introduce the response system with a controller $U \in R^{n}$ as the following equation:

$$
\begin{gathered}
\dot{y}=g(y)+\Psi(y) \Omega+D+U, \quad t \neq t_{k}, \\
\Delta y=y\left(t_{k}^{+}\right)-y\left(t_{k}^{-}\right)=y\left(t_{k}^{+}\right)-y\left(t_{k}\right)=B_{k} e, \\
t=t_{k} \quad(k=1,2, \ldots, n), \\
y\left(t_{0}^{+}\right)=y_{0},
\end{gathered}
$$

where $y \in R^{n}$ is the state vector, $y\left(t_{k}^{+}\right)=\lim _{t \rightarrow t_{k}^{+}} y(t)$, $y\left(t_{k}^{-}\right)=\lim _{t \rightarrow t_{k}^{-}} y(t), \Psi(y): R^{n} \rightarrow R^{n \times q}, \Omega \in R^{q}$ composes the unknown parameter vector of response system, $U=\left[u_{1}\right.$, $\left.u_{2}, \ldots, u_{n}\right]^{\mathrm{T}} \in R^{n}$ is the control input vector, and $B_{k}$ denotes the impulsive control gains. The impulsive instant 


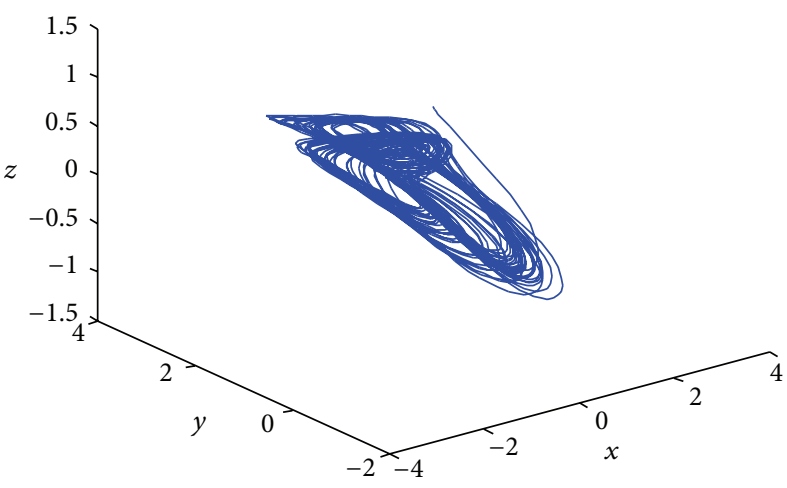

(a) $3 \mathrm{D}$ view in the $x-y-z$ space

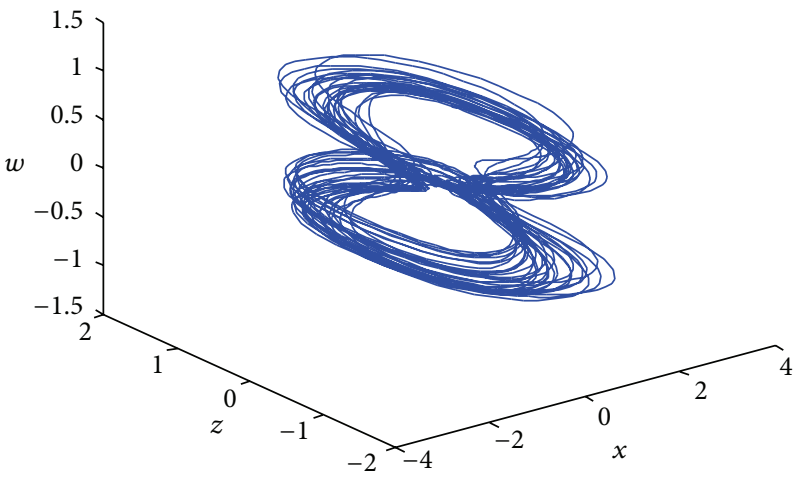

(c) 3D view in the $x-z-w$ space

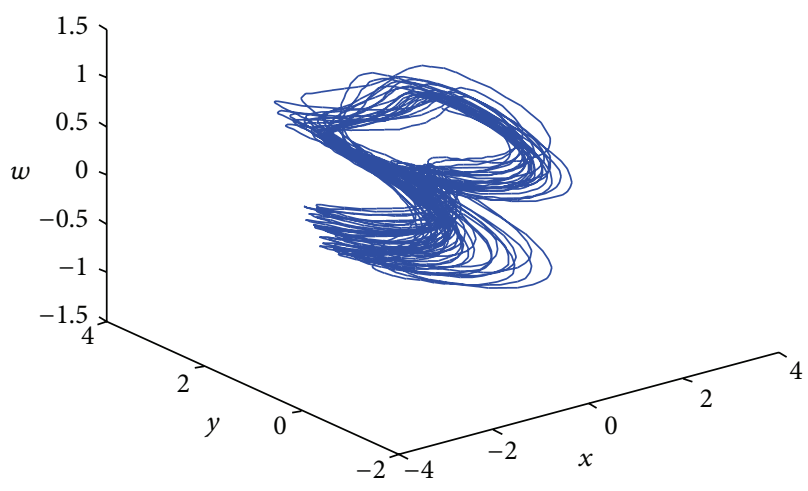

(b) $3 \mathrm{D}$ view in the $x-y-w$ space

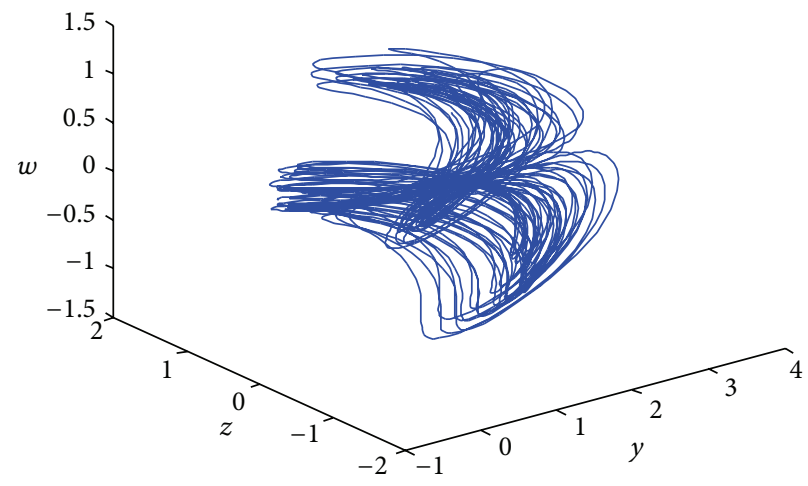

(d) $3 \mathrm{D}$ view in the $y-z-w$ space

FIGURE 2: Phase portraits of hyperchaotic finance system (1).

sequence $\left\{t_{k}\right\}_{k=1}^{+\infty}$ satisfies $0 \leq t_{1}<t_{2}<\cdots<t_{k}<t_{k+1}<\cdots$ and $\lim _{k \rightarrow \infty} t_{k}=\infty, \tau_{k}=t_{k}-t_{k-1}<\infty$.

Remark 2. Consider $D=\left[d_{1}, d_{2}, \ldots, d_{n}\right]^{\mathrm{T}} \in R^{n}$ in (3) represents the external disturbance; that is, there exists positive bounded function $\rho_{i}$ making the following inequalities hold:

$$
\left|d_{i}(t)\right| \leq \rho_{i} \quad(i=1,2, \ldots, n) .
$$

Then the synchronization error dynamic system can be obtained as follows:

$$
e(t)=\Lambda h(t) y(t)-x(t),
$$

where $\Lambda=\operatorname{diag}\left\{\Lambda_{1}, \Lambda_{2}, \ldots, \Lambda_{n}\right\}$ is a constant diagonal matrix, $h(t)=\operatorname{diag}\left\{h_{1}(t), h_{2}(t), \ldots, h_{n}(t)\right\}$, and $h_{i}(t): R^{n} \rightarrow$ $R^{n}(i=1,2, \ldots, n)$ are called scaling function factors.

Definition 3. The two systems described by system (2) and system (3) achieved modified function projective synchronization (MFPS), if there exists a continuously differentiable scaling function with $h_{i}(t) \neq 0$ and a vector controller $U$ such that

$$
\lim _{t \rightarrow \infty}\|e(t)\|=\lim _{t \rightarrow \infty}\|\Lambda h(t) y(t)-x(t)\|=0 .
$$

Remark 4. If $h_{1}(t)=h_{2}(t)=\cdots=h_{n}(t)$, the scheme of synchronization is developed to synchronize two systems to a scaling function matrix with different initial values; that is, the modified function projective synchronization problem is simplified to function projective synchronization. If $h=\operatorname{diag}\left\{h_{1}, h_{2}, \ldots, h_{n}\right\}$, where $h_{i}$ are real constants for all $i \in R$, the modified function projective synchronization converts to the modified projective synchronization. If $\Lambda h=$ $\operatorname{diag}\{1,1, \ldots, 1\}$, modified function projective synchronization converts to the complete synchronization.

From (3), we know that the error system between (2) and (3) is

$$
\begin{aligned}
\dot{e}(t)= & \Lambda h(t) \dot{y}(t)+\Lambda \dot{h}(t) y(t)-\dot{x}(t) \\
= & \Lambda h(t)[g(y)+\Psi(y) \Omega+D+U] \\
& +\Lambda \dot{h}(t) y(x)-f(x)-\Phi(x) \Theta \\
& \quad t \neq t_{k}, \quad(k=1,2, \ldots, n), \\
e\left(t_{k}^{+}\right)= & y\left(t_{k}^{+}\right)-x\left(t_{k}^{+}\right)=y\left(t_{k}\right)+B_{k} e\left(t_{k}\right)-x\left(t_{k}\right) \\
= & \left(I+B_{k}\right) e(t) \quad t=t_{k},
\end{aligned}
$$



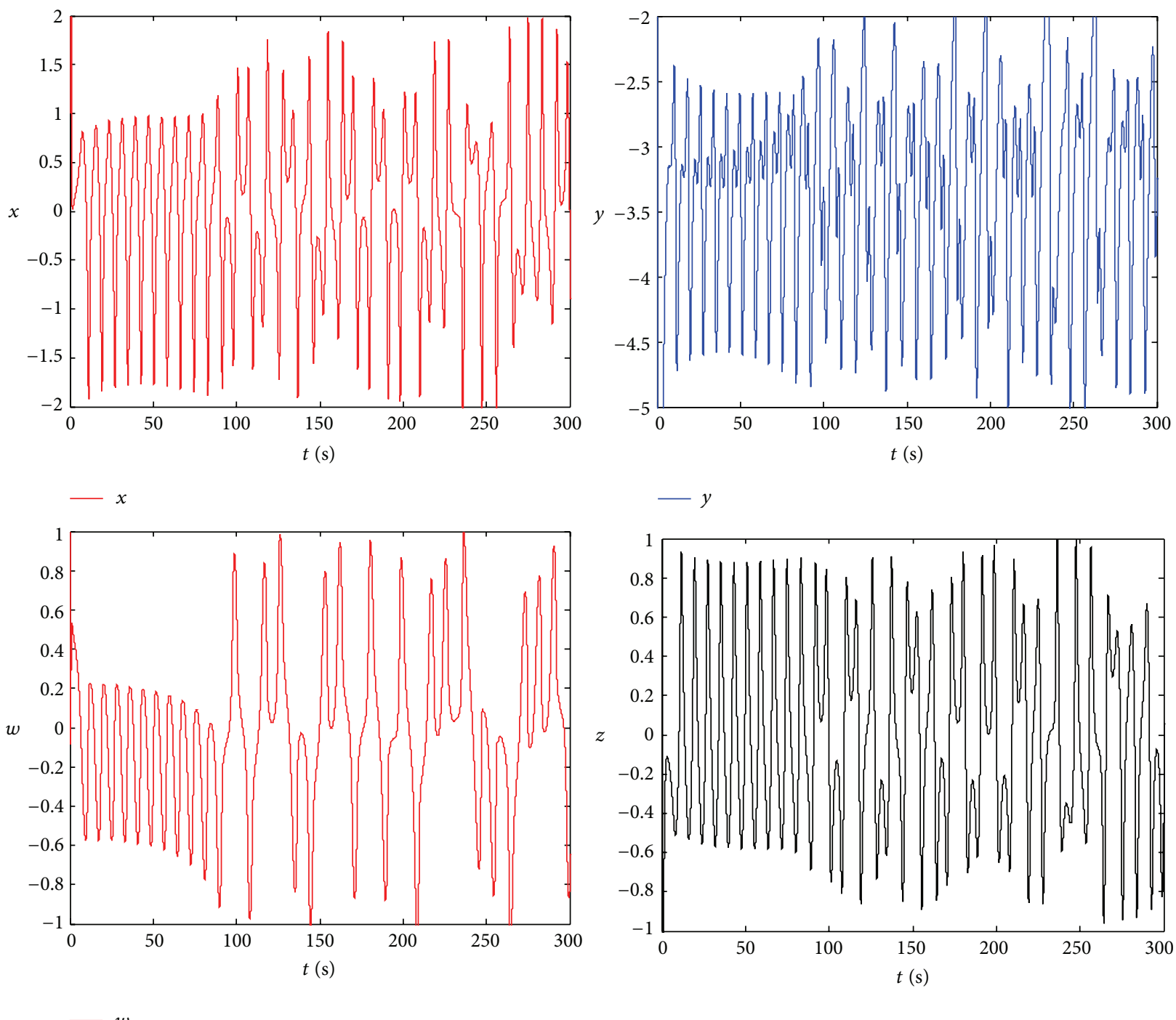

Figure 3: Time series of $(x, y, z, w)^{\mathrm{T}}$ in system (1).

where $I$ is the identical matrix with the same dimension as $B_{k}$.

For given synchronization scaling function $h_{i}(t)$ and scaling matrix $\Lambda$, the adaptive impulsive controller is as the following:

$$
\begin{gathered}
U=\frac{\Lambda^{-1}}{h(t)}[-\Lambda \dot{h}(t) y(t)+f(x)+\Phi(x) \widehat{\Theta}-m e] \\
-g(y)-\Psi(y) \widehat{\Omega}-\operatorname{sgn}\left(\operatorname { d i a g } \left\{e_{1} \Lambda_{1} h_{1}(t), e_{2} \Lambda_{2} h_{2}(t),\right.\right. \\
\left.\left.\ldots, e_{n} \Lambda_{n} h_{n}(t)\right\}\right) \rho,
\end{gathered}
$$

where $\widehat{\Theta}, \widehat{\Omega}$ are the estimations of the parameters of the two systems, $\operatorname{sgn}(\cdot)$ denotes the sign function, and $m$ is a negative constant, which would influence the rate of the convergence.
The larger $m$, the faster rate. So we can adjust the value of $m$ according to the desirable convergent rate.

The parameter update laws are as follows:

$$
\begin{gathered}
\dot{\widehat{\Theta}}=-\Phi^{\mathrm{T}}(x) e(t), \\
\dot{\widehat{\Omega}}=\Psi^{\mathrm{T}}(y) \Lambda h(t) e(t),
\end{gathered}
$$

where $\widehat{\Theta}$ and $\widehat{\Omega}$ are the estimated values of unknown parameters $\Theta$ and $\Omega$, respectively. $\widetilde{\Theta}=\Theta-\widehat{\Theta}$ and $\widetilde{\Omega}=\Omega-\widehat{\Omega}$ are estimate errors.

Remark 5. In the control law, $\operatorname{sgn}\left(\operatorname{diag}\left\{e_{1} \Lambda_{1} h(t), e_{2} \Lambda_{2} h(t)\right.\right.$, $\left.\left.\ldots, e_{n} \Lambda_{n} h(t)\right\}\right) \rho$ is the compensator that is introduced to eliminate the influence of the external uncertainties. 

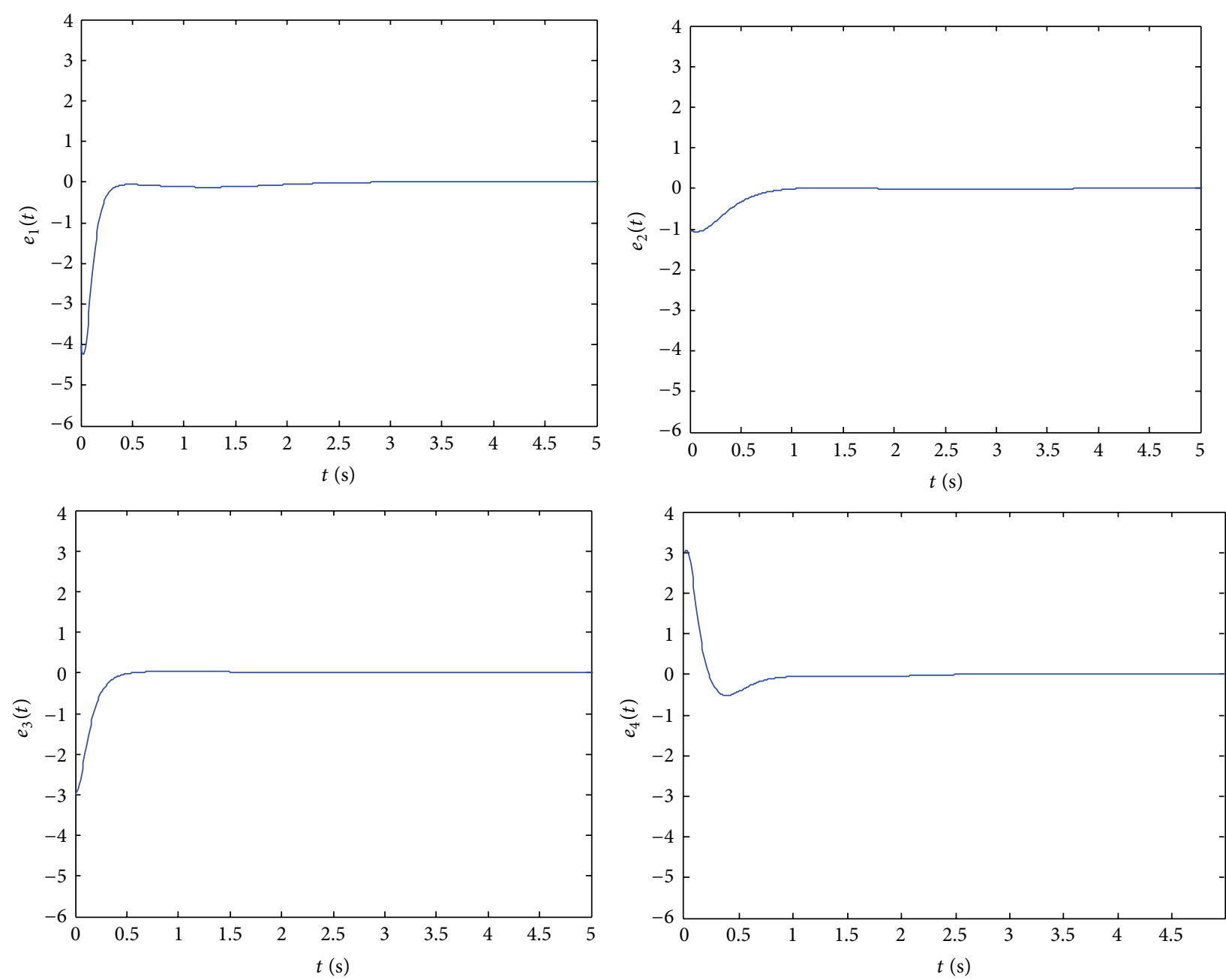

FIGURE 4: Time responses of MFPS errors with impulses.
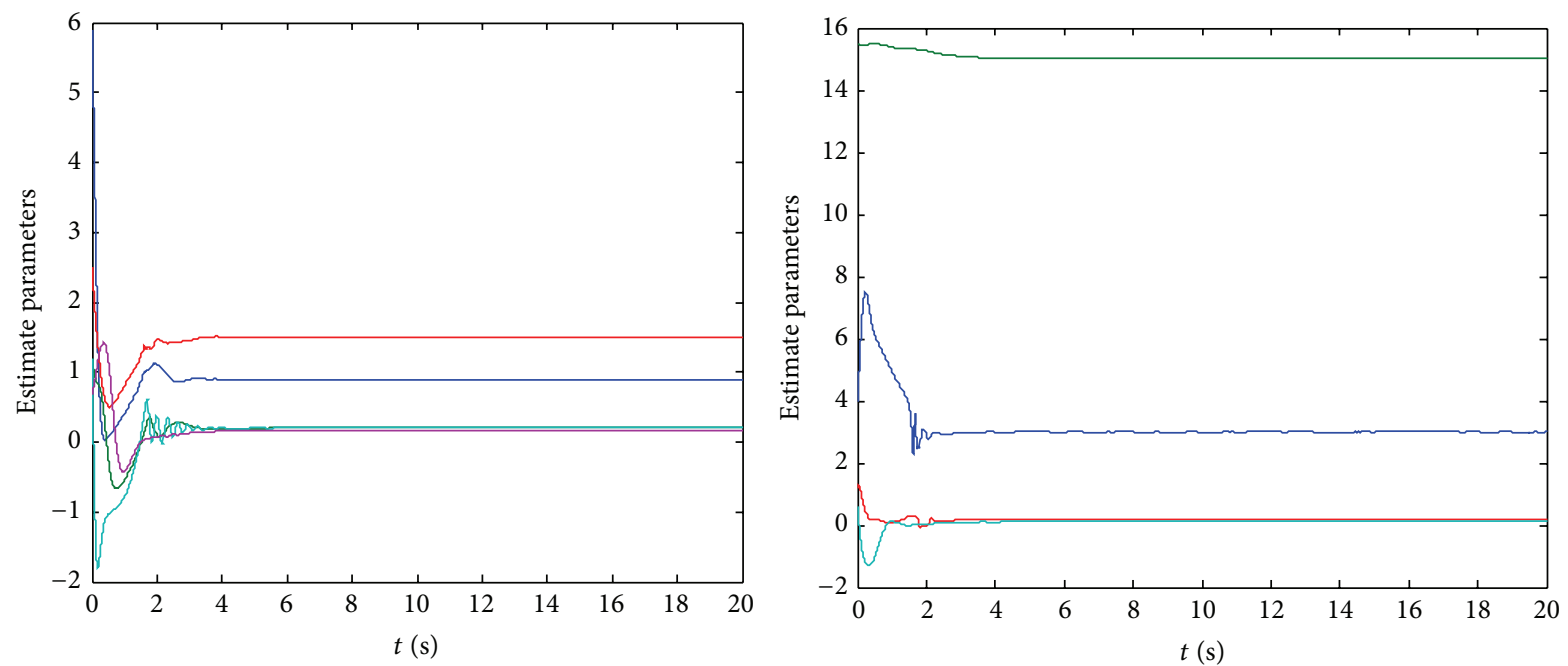
$-a_{1}$
$\begin{aligned}- & d_{1} \\ - & k_{1}\end{aligned}$
$-a_{2}$
$-c_{2}$
$-c_{1}$
$-b_{2}$
$-d_{2}$

FIGURE 5: The estimations of the unknown parameters of hyperchaotic systems. 

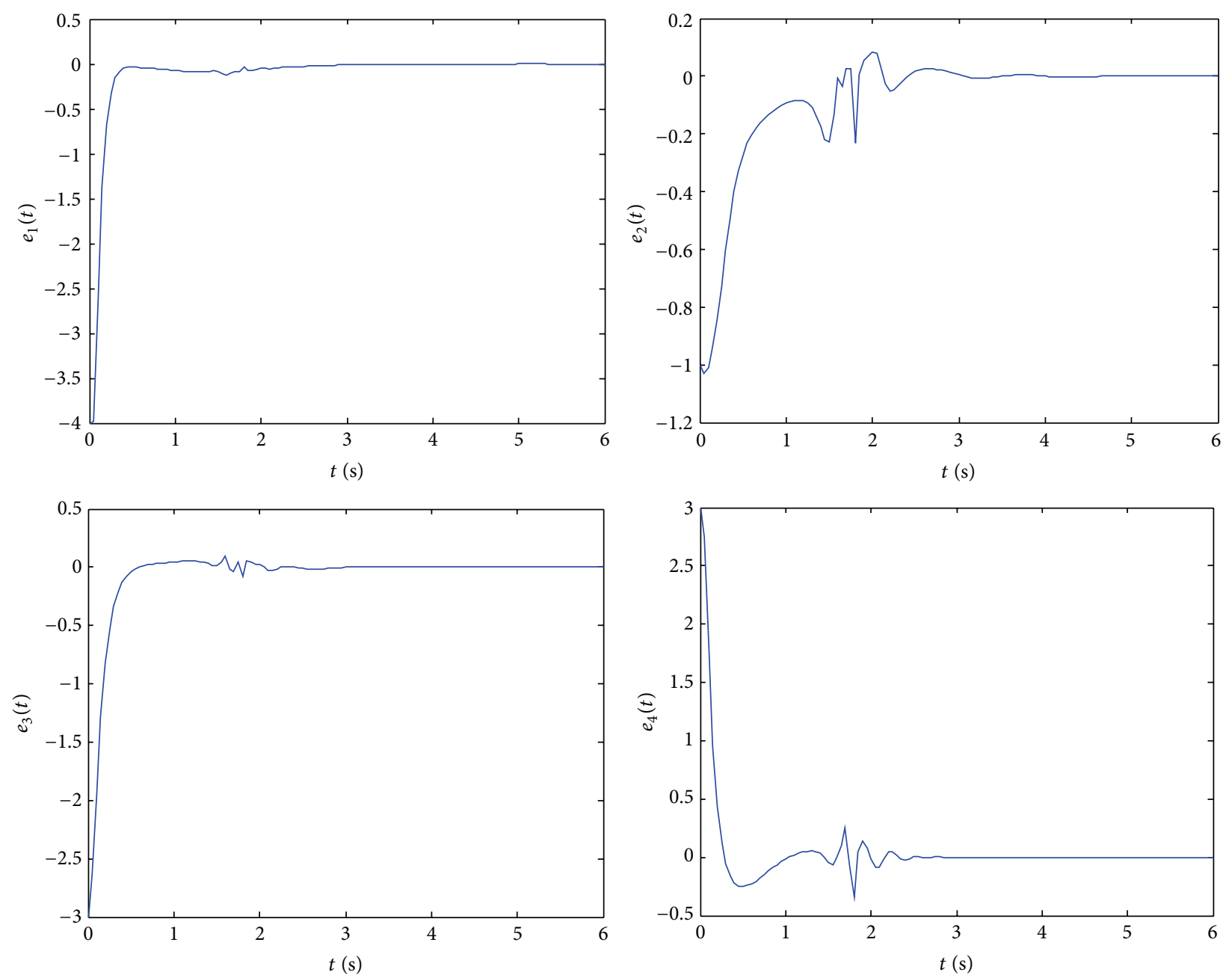

FIGURE 6: Time responses of FPS errors with impulses.

Lemma 6 (see [21]). Suppose the $q \geq 0, u(t)$ satisfies the scalar impulsive different inequality:

$$
\begin{gathered}
D^{+} u(t) \leq-p u(t)+q\left(\sup _{t-\tau \leq s \leq t} u(s)\right), \quad t \neq t_{k}, t \geq t_{0}, \\
u\left(t_{k}\right) \leq \alpha_{k} u\left(t_{k}^{-}\right), \quad 0<\alpha_{k-1} \leq 1, k \in Z, \\
u(t)=\phi(t), \quad t \in\left[t_{0}-\tau, t_{0}\right],
\end{gathered}
$$

where $u(t)$ is continuous at $t \neq t_{k}, t \geq t_{0}$ and $u\left(t_{k}\right)=u\left(t_{k}^{+}\right)$and $u\left(t_{k}^{-}\right)$exist. Consider $\phi \in P C\left(\left[t_{0}-\tau, t_{0}\right], R\right)$. And the following condition is satisfied:

$$
\left(-p+\frac{q}{\alpha_{k-1}}\right)\left(t_{k}-t_{k-1}\right)<-\ln \alpha_{k-1}, \quad k \in Z^{+} .
$$

Then error system (7) is asymptotically stable.

Now, the goal is to find some conditions on the control gains. Because the impulsive interval $\tau_{k}=t_{k}-t_{k-1}=\tau$ is a positive constant, the drive system is synchronized with the response system for arbitrary initial conditions. Thus we can present the following theorem.
Theorem 7. System (2) and system (3) can realize MFPS under adaptive impulse controller (8) and updated laws of the parameters (9) and (10) and satisfy the following conditions:

$$
\begin{aligned}
& \text { (1) } \Delta_{k}=\sup _{k}\left\{\tau_{k}-\tau_{k-1}\right\}<\infty, \\
& \text { (2) } \alpha_{k}=\lambda_{\max }\left[\left(I+B_{k}\right)^{\mathrm{T}}\left(I+B_{k}\right)\right]<1, \\
& \text { (3) }\left(-p+q / \alpha_{k-1}\right)\left(t_{k}-t_{k-1}\right)<-\ln \alpha_{k-1}, k \in Z^{+},
\end{aligned}
$$

where $\lambda_{\max }(\cdot)$ is the maximum eigenvalue.

Proof. Let us choose the following Lyapunov function:

$$
V=\frac{1}{2}\left(e^{\mathrm{T}} e+\widetilde{\Theta}^{\mathrm{T}} \widetilde{\Theta}+\widetilde{\Omega}^{\mathrm{T}} \widetilde{\Omega}\right) .
$$

With the controllers and the parameter update laws, here noting $\rho=\left[\rho_{1}, \rho_{2}, \ldots, \rho_{n}\right]^{\mathrm{T}}$, the time derivation of the Lyapunov 
function along the trajectory of error system is given in two cases. In the case when $t \neq t_{k}$, we have

$$
\begin{aligned}
& \dot{V}= e^{\mathrm{T}} \dot{e}+\widetilde{\Theta}^{\mathrm{T}} \widetilde{\Theta}+\widetilde{\Omega}^{\mathrm{T}} \widetilde{\Omega} \\
&= e^{\mathrm{T}}\{\Lambda h(t)[g(y)+\Psi(y) \Omega+D+U] \\
&+\Lambda \dot{h}(t) y(x)-f(x)-\Phi(x) \Theta\} \\
&+(\Theta-\widehat{\Theta})^{\mathrm{T}}(-\dot{\widehat{\Theta}})+(\Omega-\widehat{\Omega})^{\mathrm{T}}(-\dot{\widehat{\Omega}}) \\
&=-m e^{\mathrm{T}} e+e^{\mathrm{T}} \Lambda h(t) \\
& \cdot\left[D-\operatorname{sgn}\left(\operatorname { d i a g } \left\{e_{1} \Lambda_{1} h(t), e_{2} \Lambda_{2} h(t), \ldots,\right.\right.\right. \\
&\left.\left.\left.\quad e_{n} \Lambda_{n} h(t)\right\}\right) \rho\right] \\
&=-m e^{\mathrm{T}} e+h(t) e^{\mathrm{T}} \Lambda D \quad \\
&-h(t) e^{\mathrm{T}} \Lambda \operatorname{sgn}\left(\operatorname { d i a g } \left\{e_{1} \Lambda_{1} h(t), e_{2} \Lambda_{2} h(t), \ldots,\right.\right. \\
&\left.\left.e_{n} \Lambda_{n} h(t)\right\}\right) \rho .
\end{aligned}
$$

In the case when $t=t_{k}$, we have

$$
\begin{aligned}
V\left(t_{k}^{+}\right)= & \frac{1}{2} e^{\mathrm{T}}\left(t_{k}^{+}\right) e\left(t_{k}\right)+\frac{1}{2}\left(\widetilde{\alpha}^{\mathrm{T}} \widetilde{\alpha}+\widetilde{\beta}^{\mathrm{T}} \widetilde{\beta}\right) \\
= & \frac{1}{2}\left[\left(1+B_{i}\right) e_{k}\right]^{\mathrm{T}}\left[\left(1+B_{i}\right) e_{k}\right]+\frac{1}{2}\left(\widetilde{\alpha}^{\mathrm{T}} \widetilde{\alpha}+\widetilde{\beta}^{\mathrm{T}} \widetilde{\beta}\right) \\
\leq & \frac{1}{2} \lambda_{\max }\left[\left(I+B_{i}\right)^{\mathrm{T}}\left(I+B_{i}\right)\right] e^{\mathrm{T}}\left(t_{k}\right) e\left(t_{k}\right) \\
& +\frac{1}{2}\left(\widetilde{\alpha}^{\mathrm{T}} \widetilde{\alpha}+\widetilde{\beta}^{\mathrm{T}} \widetilde{\beta}\right) \\
\leq & \alpha_{k} V\left(t_{k}\right),
\end{aligned}
$$

where $\alpha_{k}=\lambda_{\text {max }}\left[\left(I+B_{k}\right)^{\mathrm{T}}\left(I+B_{k}\right)\right]<1$.

Let $\xi_{1}=h(t) e^{\mathrm{T}} \Lambda D, \xi_{2}=h(t) e^{\mathrm{T}} \Lambda \operatorname{sgn}\left(\operatorname{diag}\left\{e_{1} \Lambda_{1} h(t)\right.\right.$, $\left.\left.e_{2} \Lambda_{2} h(t), \ldots, e_{n} \Lambda_{n} h(t)\right\}\right) \rho$. We see that $\xi_{1}, \xi_{2} \in R$ and $\xi_{2} \geq 0$. According to the definition and assumption of $\rho$ and $D$, it is guaranteed that $\xi_{1}-\xi_{2} \leq 0$; therefore it can be obtained that

$$
\dot{V}(t)=-m e^{\mathrm{T}} e+\xi_{1}-\xi_{2} \leq-m e^{\mathrm{T}} e \leq-2 m V(t),
$$

where $2 m=p$; then $\dot{V}(t) \leq-p V(t)$.

Then, we can obtain the following comparison system:

$$
\begin{gathered}
D^{+} u(t) \leq-p u(t)+q\left(\sup _{t-\tau \leq s \leq t} u(s)\right), \quad t \neq t_{k}, \quad t \geq t_{0}, \\
u\left(t_{k}\right) \leq \alpha_{k} u\left(t_{k}^{-}\right), \quad 0<\alpha_{k-1}<1, k \in Z, \\
u(t)=\phi(t), \quad t \in\left[t_{0}-\tau, t_{0}\right] .
\end{gathered}
$$

When $q=0, \alpha_{k}=\alpha_{k-1}<1$, and $\left(-p+q / \alpha_{k-1}\right)\left(t_{k}-t_{k-1}\right)<$ $-\ln \alpha_{k-1}, k \in Z^{+}$, the stability conditions in comparison theorem are satisfied. We know that the error system is asymptotically stable, that is, the synchronization of the chaotic system under the impulsive controllers.

The proof is completed.

Based on Lyapunov stability theory, we discussed MFPS between the drive and response system. By using the impulsive control, this drive-response hyperchaotic system can easily be synchronized. Some sufficient conditions for impulsive synchronization are derived. So we present this method to control financial system.

\section{MFPS of the Financial Hyperchaotic Systems with Uncertain Parameters}

In this section, new financial hyperchaotic system (1) will be adopted to verify the effectiveness of the MFPS schemes with the parameter identification obtained in Section 3.

To further illustrate the effectiveness of the controller, we select the new financial hyperchaotic system as the drive system:

$$
\begin{aligned}
& \dot{x}_{1}=x_{3}+\left(x_{2}-a_{1}\right) x_{1}+x_{4}, \\
& \dot{x}_{2}=1-b_{1} x_{2}-x_{1}^{2}, \\
& \dot{x}_{3}=-x_{1}-c_{1} x_{3}, \\
& \dot{x}_{4}=-d_{1} x_{1} x_{2}-k_{1} x_{4},
\end{aligned}
$$

where $u_{1}, u_{2}, u_{3}$, and $u_{4}$ are controllers to be designed and $a$, $b, c, d$, and $k$ are unknown parameters.

System (18) can be presented in the form of

$$
\left(\begin{array}{c}
\dot{x}_{1} \\
\dot{x}_{2} \\
\dot{x}_{3} \\
\dot{x}_{4}
\end{array}\right)=\left(\begin{array}{c}
x_{3}+x_{1} x_{2}+x_{4} \\
1-x_{1}^{2} \\
-x_{1} \\
0
\end{array}\right)
$$

$$
+\left(\begin{array}{ccccc}
-x_{1} & 0 & 0 & 0 & 0 \\
0 & -x_{2} & 0 & 0 & 0 \\
0 & 0 & -x_{3} & 0 & 0 \\
0 & 0 & 0 & -x_{1} x_{2} & -x_{4}
\end{array}\right)\left(\begin{array}{c}
a_{1} \\
b_{1} \\
c_{1} \\
d_{1} \\
k_{1}
\end{array}\right) .
$$

We choose the novel hyperchaotic system [22] as the response system:

$$
\begin{aligned}
& \dot{y}_{1}=-a_{2}\left(y_{1}+y_{2}\right)+y_{4}+d_{1}+u_{1}, \\
& \dot{y}_{2}=-y_{2}-a_{2} y_{1} y_{3}+d_{2}+u_{2}, \\
& \dot{y}_{3}=b_{2}+a_{2} y_{1} y_{2}+d_{3}+u_{3}, \\
& \dot{y}_{4}=-c_{2} y_{1} y_{3}-d_{2} y_{4}+d_{4}+u_{4},
\end{aligned}
$$

supposing all the parameters in the two systems are unknown. 

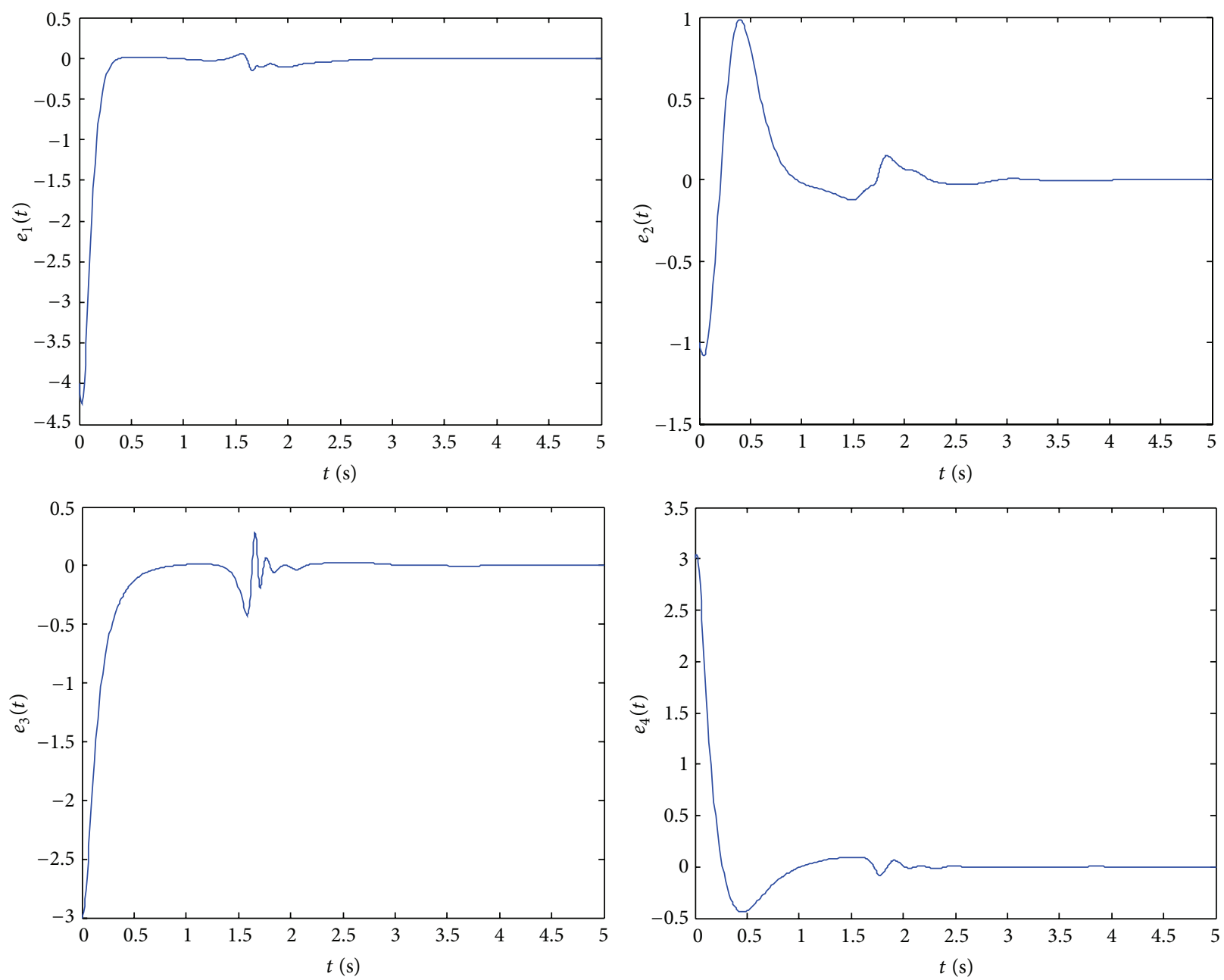

FIGURE 7: Time responses of FPS errors without impulses.

Then the above system can be described in the form of

$$
\begin{aligned}
\left(\begin{array}{l}
\dot{y}_{1} \\
\dot{y}_{2} \\
\dot{y}_{3} \\
\dot{y}_{4}
\end{array}\right)= & \left(\begin{array}{c}
y_{4} \\
-y_{2} \\
0 \\
0
\end{array}\right) \\
& +\left(\begin{array}{cccc}
-y_{1}-y_{2} & 0 & 0 & 0 \\
-y_{1} y_{3} & 0 & 0 & 0 \\
y_{1} y_{2} & 1 & 0 & 0 \\
0 & 0 & -y_{1} y_{3} & -y_{4}
\end{array}\right)\left(\begin{array}{l}
a_{2} \\
b_{2} \\
c_{2} \\
d_{2}
\end{array}\right) \\
& +\left(\begin{array}{c}
d_{1} \\
d_{2} \\
d_{3} \\
d_{4}
\end{array}\right)+\left(\begin{array}{l}
u_{1} \\
u_{2} \\
u_{3} \\
u_{4}
\end{array}\right) .
\end{aligned}
$$

According to Theorem 7, the controllers can be obtained as

$$
\begin{aligned}
u_{1}= & \frac{1}{\Lambda_{1} h_{1}(t)}\left[x_{3}+\left(x_{2}-\widehat{a}_{1}\right) x_{1}+x_{4}-\Lambda_{1} \dot{h}_{1}(t) y_{1}-m e_{1}\right] \\
& +\widehat{a}_{2}\left(y_{1}+y_{2}\right)-y_{4}-\rho_{1} \operatorname{sgn}\left(\Lambda_{1} h(t) e_{1}\right), \\
u_{2}= & \frac{1}{\Lambda_{2} h_{2}(t)}\left[1-\widehat{b}_{1} x_{2}-x_{1}^{2}-\Lambda_{2} \dot{h}_{2}(t) y_{2}-m e_{2}\right] \\
& +y_{2}+\widehat{a}_{2} y_{1} y_{3}-\rho_{2} \operatorname{sgn}\left(\Lambda_{2} h(t) e_{2}\right), \\
u_{3}= & \frac{1}{\Lambda_{3} h_{3}(t)}\left[-x_{1}-\widehat{c}_{1} x_{3}-\Lambda_{3} \dot{h}_{3}(t) y_{3}-m e_{3}\right] \\
& -\widehat{b}_{2}-\widehat{a}_{2} y_{1} y_{2}-\rho_{3} \operatorname{sgn}\left(\Lambda_{3} h(t) e_{3}\right), \\
u_{4}= & \frac{1}{\Lambda_{4} h_{4}(t)}\left[-\widehat{d}_{1} x_{1} x_{2}-\widehat{k}_{1} x_{4}-\Lambda_{4} \dot{h}_{4}(t) y_{4}-m e_{4}\right] \\
& +\widehat{c}_{2} y_{1} y_{3}+\widehat{d}_{2} y_{4}-\rho_{4} \operatorname{sgn}\left(\Lambda_{4} h(t) e_{4}\right) .
\end{aligned}
$$



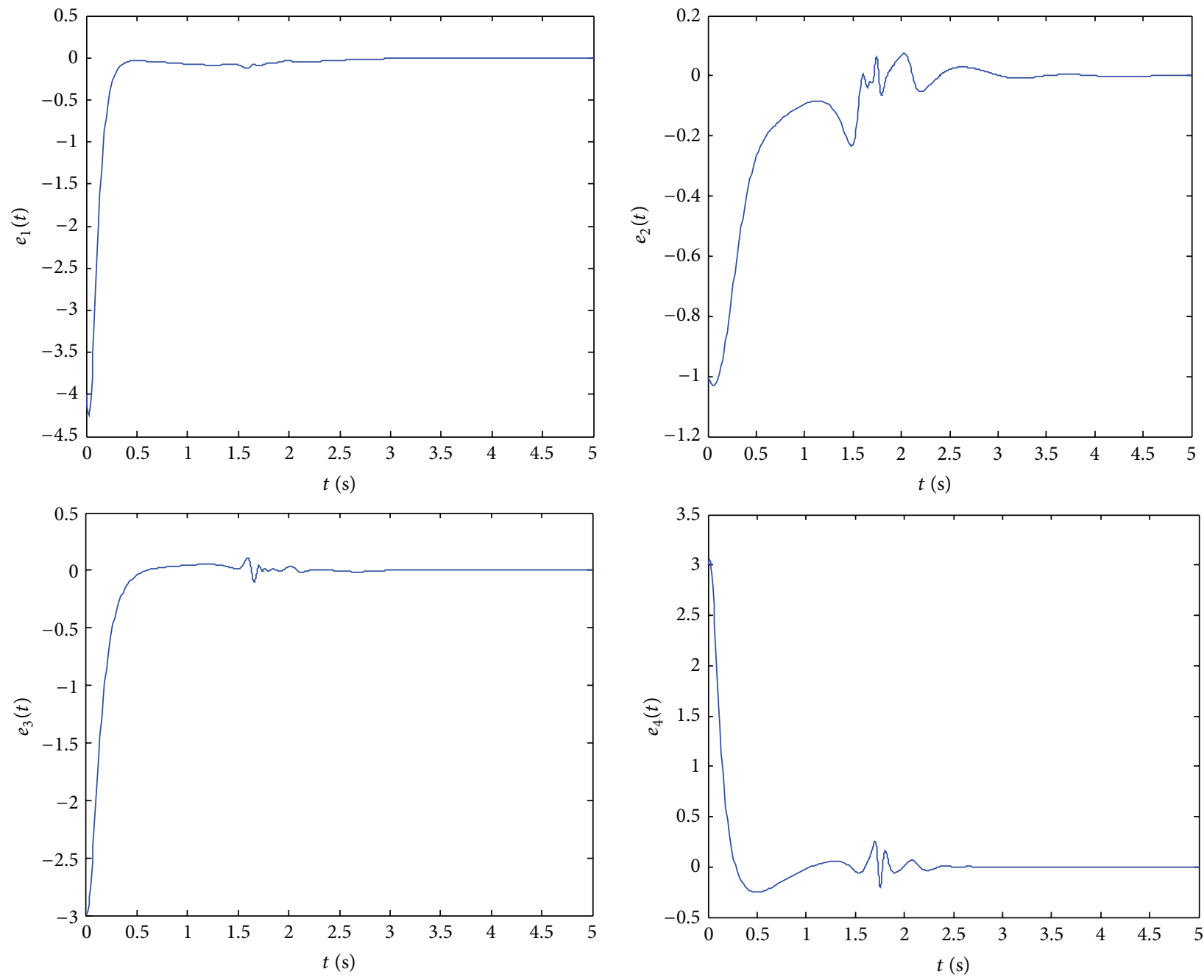

FIGURE 8: Time responses of MFPS errors without impulses.

And the parameters estimation update laws are given as

$$
\begin{gathered}
\dot{\hat{a}}_{1}=x_{1} e_{1}, \quad \dot{\hat{b}}_{1}=x_{2} e_{2}, \quad \dot{\hat{c}}_{1}=x_{3} e_{3}, \\
\dot{\hat{d}}_{1}=x_{1} x_{2} e_{4}, \quad \dot{\hat{k}}_{1}=x_{4} e_{4}, \\
\dot{\hat{a}}_{2}=-\left(y_{1}+y_{2}\right) \Lambda_{1} h(t) e_{1}-y_{1} y_{3} \Lambda_{2} h(t) e_{2} \\
+y_{1} y_{2} \Lambda_{3} h(t) e_{3}, \\
\dot{\hat{b}}_{2}=\Lambda_{3} h(t) e_{3}, \quad \dot{\hat{c}}_{2}=-y_{1} y_{3} \Lambda_{4} h(t) e_{4}, \\
\dot{\hat{d}}_{2}=-y_{4} \Lambda_{4} h(t) e_{4} .
\end{gathered}
$$

Through the above results, error system can be asymptotically stable.

\section{Numerical Simulations}

To verify the results obtained in Section 4, some numerical simulations will be performed in this section. We choose system (18) as the drive system and system (20) as the response system. The parameters are chosen as $a_{1}=0.9, b_{1}=0.2, c_{1}=$ 1.5, $d_{1}=0.2, a_{2}=3, b_{2}=15, c_{2}=0.2, d_{2}=0.12$, and $k_{2}=$ 0.17 . The initial values of the drive system and the response system are $\left(x_{1}(0), x_{2}(0), x_{3}(0), x_{4}(0)\right)=(-0.5,-5,-5,-4)$ and $\left(y_{1}(0), y_{2}(0), y_{3}(0), y_{4}(0)\right)=(-4,-2,-2,-1)$. And the external disturbances are supposed as $d_{1}=0.5 \cos (2 \pi t), d_{2}=$ $0.2 \sin (5 \pi t), d_{3}=0.5 \cos (6 \pi t)$, and $d_{4}=0.2 \sin (5 \pi t)$. Thus the boundaries of the uncertainties are $\rho_{1}=\rho_{2}=\rho_{3}=\rho_{4}=1$. The scaling matrix is taken as $\Lambda=\operatorname{diag}\{1,-1,-1,-1\}$, while the scaling function is taken as $h_{1}(t)=\sin (t), h_{2}(t)=\cos (t)$, $h_{3}(t)=\sin (t)$, and $h_{4}(t)=\cos (t)$. And choose $m=-1$, $p=-2, \tau=0.01, B_{k}=\operatorname{diag}\{-0.9,-0.9,-0.9,-0.9\}$, and $\alpha_{k}=$ $\lambda_{\text {max }}\left[\left(I+B_{k}\right)^{\mathrm{T}}\left(I+B_{k}\right)\right]=0.01<1$; the value of the parameters has obtained the conditions in Theorem 7 . The initial values of the unknown parameters are as $\dot{\widehat{a}}_{1}=\dot{\hat{b}}_{1}=\dot{\widehat{c}}_{1}=\dot{\vec{d}}_{1}=\dot{\hat{k}}_{1}=$ $1, \dot{\hat{a}}_{2}=\dot{\widehat{b}}_{2}=\dot{\widehat{c}}_{2}=\dot{\hat{d}}_{2}=1$. Assume the controllers are switched on; then $t=0 \mathrm{~s}$. Using MATLAB, we get the time responses of MFPS errors with impulses in Figure 4 and the estimations of the unknown parameters of hyperchaotic systems in Figure 5. 
In order to illustrate the superiority of MFPS method, we let the drive system, response system with impulses, parameters, and initial values be the same as before. The time responses of FPS errors with impulses are shown in Figure 6. Comparing Figure 4 with Figure 6, we can observe two facts about the errors of MFPS method with impulse compared to the errors of FPS method with impulse: first, they converge to zero faster; second, their fluctuation is smaller.

Considering the traditional FPS method without impulse control, we let the drive system, response system without impulse, parameters, and initial values be the same as before. The time responses of FPS errors without impulses are given in Figure 7. The time responses of MFPS errors without impulses are shown in Figure 8.

From Figures 7 and 8, it is easy to find that the errors of MFPS without impulse have smaller fluctuation than those of FPS without impulse. This proved that the MFPS method is better than the FPS method. To further explain the advantages of MFPS method, we compare Figure 4 with Figure 7. It is obvious that the response and drive system synchronize in a shorter time under the MFPS method with impulse control than the FPS method without impulse. Those above results show the correctness and effectiveness of our methods via impulsive controller. As we all know, hyperchaotic financial system (18) is just one of the hyperchaotic systems. Furthermore, the MFPS method is a generalized form of FPS method from Definition 3 and Remark 2. So the MFPS method has wide application and can be applied to many other hyperchaotic systems such as hyperchaotic Chen system and hyperchaotic Lorenz system.

In a summary, the MFPS method has three advantages: quick convergence, smaller fluctuation, and wide application. The MFPS method consuming more energy under impulse is a disadvantage.

Remark 8 (the practical meaning of MFPS method). There exist different levels of financial development in different regions. However, after using MFPS method, that is, taking some measures to promote the controlled financial system, it can make two different financial systems of different levels achieve synchronization level finally.

\section{Conclusion}

In this paper, we have introduced a new financial hyperchaotic system. Then we studied its MFPS method via an impulsive control. Through this paper, some less conservative conditions for its MFPS method are obtained. In the case of the equal impulsive distances, we use some simple and easily verified sufficient conditions to guarantee MFPS method. At last, simulated examples have been presented to show the effectiveness of the proposed impulsive control scheme.

\section{Conflict of Interests}

The authors declare that there is no conflict of interests regarding the publication of this paper.

\section{Acknowledgments}

This work was supported by the National Nature Science Foundation of China (Grants 51276081 and 11171135), the Society Science Foundation from Ministry of Education of China (Grants 12YJAZH002 and 08JA790057), the Priority Academic Program Development of Jiangsu Higher Education Institutions, the Advanced Talents' Foundation of Jiangsu University (Grants 07JDG054 and 10JDG140), and the Students' Research Foundation of Jiangsu University (no. Y13A126). In particular, thanks are due to the support of Jiangsu University.

\section{References}

[1] T. Yang and L. O. Chua, "Secure communication via chaotic parameter modulation," IEEE Transactions on Circuits and Systems I: Fundamental Theory and Applications, vol. 43, no. 9, pp. 817-819, 1996.

[2] C. Li, X. Liao, and K. W. Wong, "Chaotic lag synchronization of coupled time-delayed systems and its applications in secure communication," Physica D. Nonlinear Phenomena, vol. 194, no. 3-4, pp. 187-202, 2004.

[3] M. Feki, "An adaptive chaos synchronization scheme applied to secure communication," Chaos, Solitons and Fractals, vol. 18, no. 1, pp. 141-148, 2003.

[4] L. M. Pecora and T. L. Carroll, "Synchronization in chaotic systems," Physical Review Letters, vol. 64, no. 8, pp. 821-824, 1990.

[5] G. L. Cai, S. Zheng, and L. X. Tian, "Adaptive control and synchronization of an uncertain new hyperchaotic Lorenz system," Chinese Physics B, vol. 17, no. 7, pp. 2412-2419, 2008.

[6] M. G. Rosenblum, A. S. Pikovsky, and J. Kurths, "Phase synchronization of chaotic oscillators," Physical Review Letters, vol. 76, no. 11, pp. 1804-1807, 1996.

[7] J. M. González-Miranda, "Generalized synchronization in directionally coupled systems with identical individual dynamics," Physical Review E-Statistical, Nonlinear, and Soft Matter Physics, vol. 65, no. 4, Article ID 047202, 2002.

[8] G. Cai, P. Hu, and Y. Li, "Modified function lag projective synchronization of a financial hyperchaotic system," Nonlinear Dynamics, vol. 69, no. 3, pp. 1457-1464, 2012.

[9] J. Yu, C. Hu, H. Jiang, and Z. Teng, "Synchronization of nonlinear systems with delays via periodically nonlinear intermittent control," Communications in Nonlinear Science and Numerical Simulation, vol. 17, no. 7, pp. 2978-2989, 2012.

[10] G. Cai, H. Wang, and S. Zheng, "Adaptive function projective synchronization of two different hyperchaotic systems with unknown parameters," Chinese Journal of Physics, vol. 47, no. 5, pp. 662-669, 2009.

[11] X. F. Wu, C. Xu, J. W. Feng, Y. Zhao, and X. Zhou, "Generalized projective synchronization between two different neural networks with mixed time delays," Discrete Dynamics in Nature and Society, vol. 2012, Article ID 153542, 19 pages, 2012.

[12] H. J. Liu, G. Q. Liu, H. Yu, and Z. L. Zhu, "Modified projective outer synchronization between two fractional order complex networks via adaptive control," Discrete Dynamics in Nature and Society, vol. 2014, Article ID 580378, 8 pages, 2014.

[13] Y. G. Ma and Z. J. Dong, "Finite-time adaptive synchronization of a new hyperchaotic system with uncertain parameters," Mathematical Problems in Engineering, vol. 2014, Article ID 162739, 7 pages, 2014. 
[14] S. Zheng, G. G. Dong, and Q. S. Bi, "Adaptive modified function projective synchronization of hyperchaotic systems with unknown parameters," Communications in Nonlinear Science and Numerical Simulation, vol. 15, no. 11, pp. 3547-3556, 2010.

[15] Z. B. Li and X. S. Zhao, "Generalized function projective synchronization of two different hyperchaotic systems with unknown parameters," Nonlinear Analysis. Real World Applications, vol. 12, no. 5, pp. 2607-2615, 2011.

[16] H. Y. Du, Q. S. Zeng, and C. H. Wang, "Modified function projective synchronization of chaotic system," Chaos, Solitons and Fractals, vol. 42, no. 4, pp. 2399-2404, 2009.

[17] G. Fu, "Robust adaptive modified function projective synchronization of different hyperchaotic systems subject to external disturbance," Communications in Nonlinear Science and Numerical Simulation, vol. 17, no. 6, pp. 2602-2608, 2012.

[18] H. J. Yu, G. L. Cai, and Y. X. Li, "Dynamic analysis and control of a new hyperchaotic finance system," Nonlinear Dynamics, vol. 67, no. 3, pp. 2171-2182, 2012.

[19] C. Hu and H. J. Jiang, "Time-delayed impulsive control of chaotic system based on T-S fuzzy model," Mathematical Problems in Engineering, vol. 2014, 12 pages, 2014.

[20] C. Li, X. Liao, and X. Zhang, "Impulsive synchronization of chaotic systems," Chaos, vol. 15, no. 2, Article ID 023104, 2005.

[21] J. Zhou, Q. j. Wu, L. Xiang, S. M. Cai, and Z. R. Liu, "Impulsive synchronization seeking in general complex delayed dynamical networks," Nonlinear Analysis. Hybrid Systems, vol. 5, no. 3, pp. 513-524, 2011.

[22] J. Ding, W. Yang, and H. Yao, "A new modified hyperchaotic finance system and its control," International Journal of Nonlinear Science, vol. 8, no. 1, pp. 59-66, 2009. 


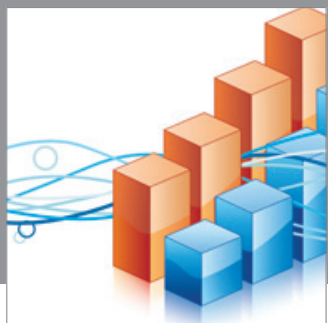

Advances in

Operations Research

mansans

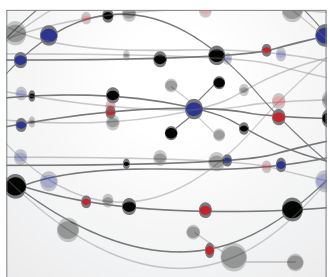

The Scientific World Journal
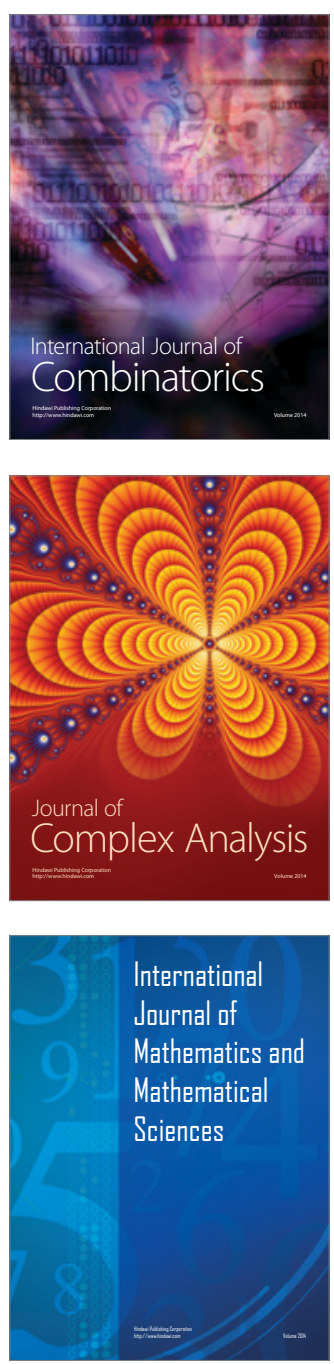
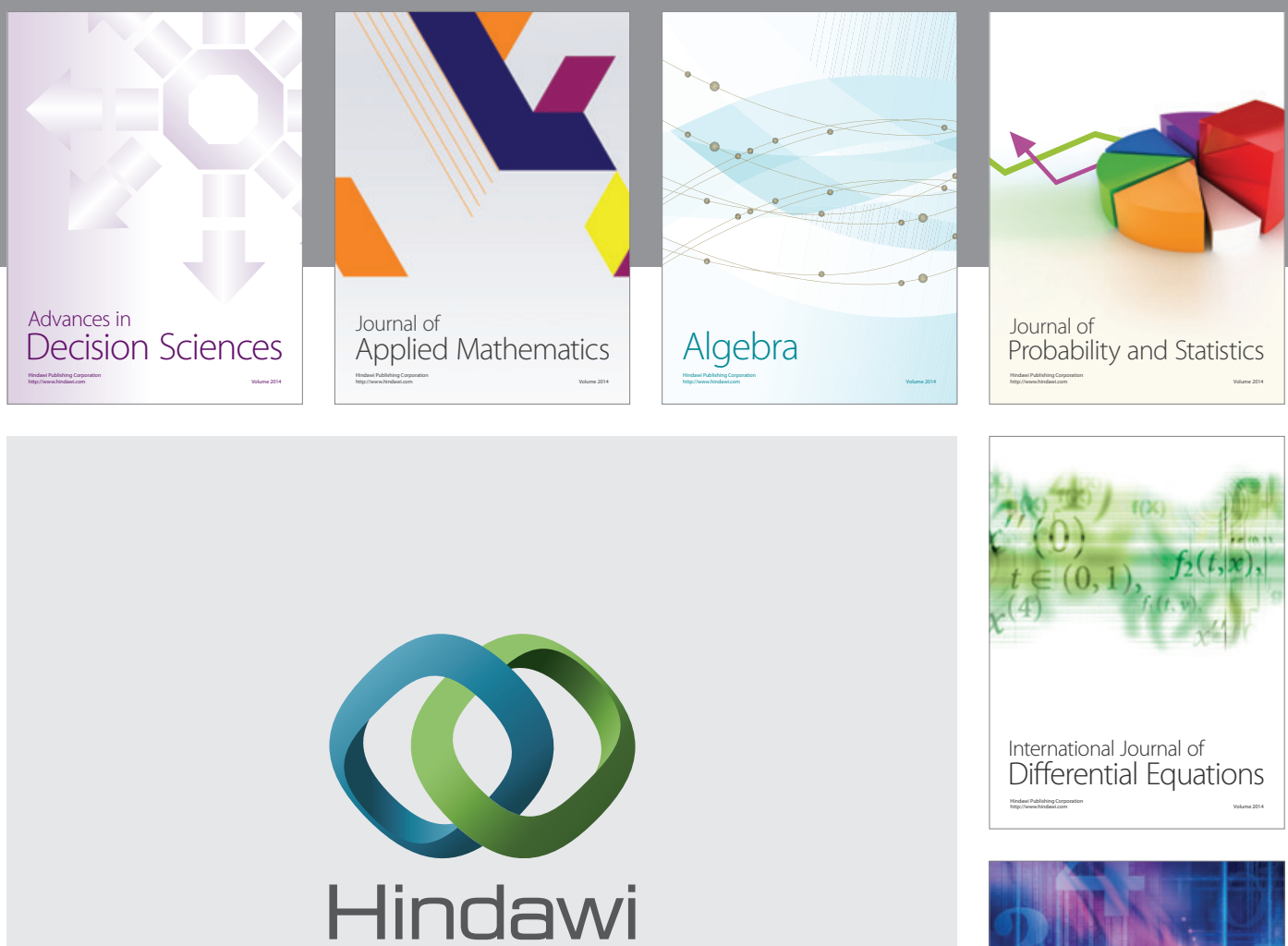

Submit your manuscripts at http://www.hindawi.com
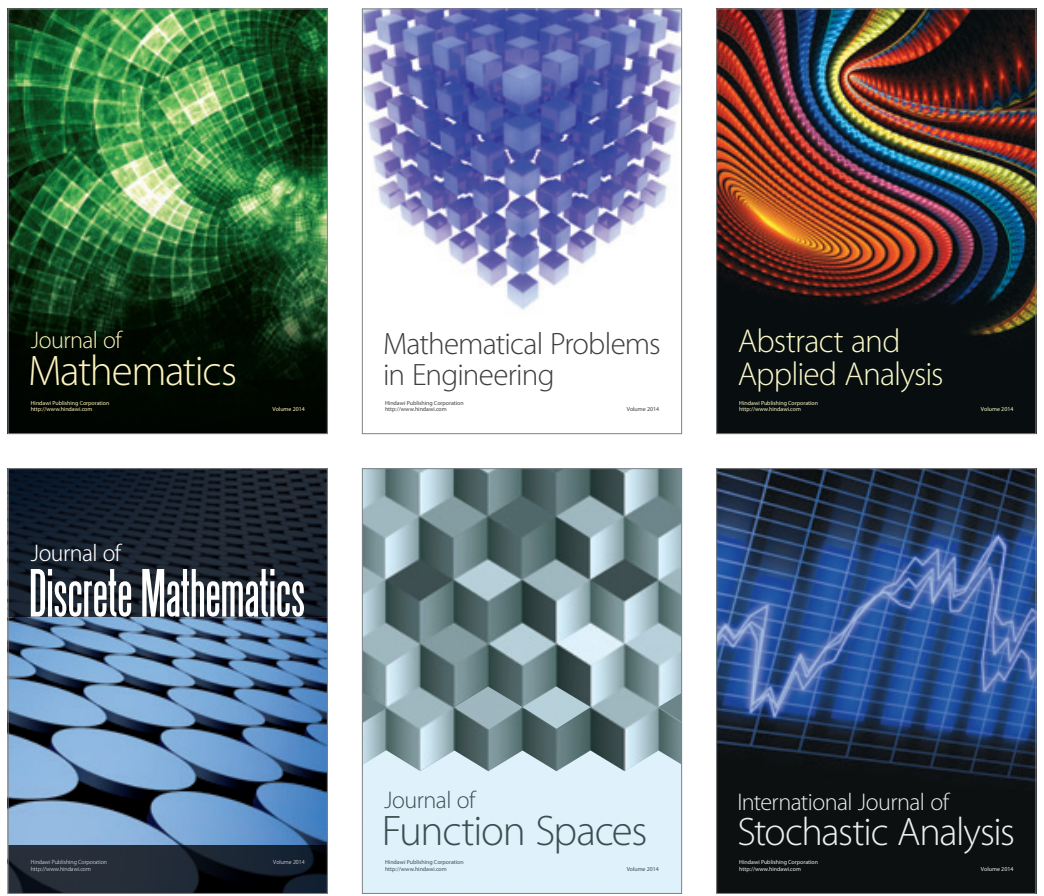

Journal of

Function Spaces

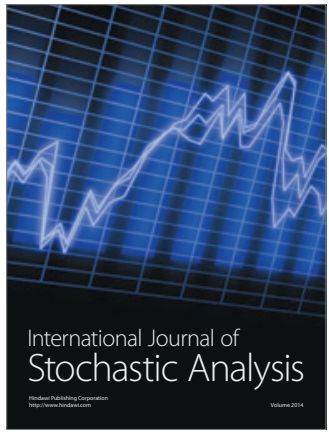

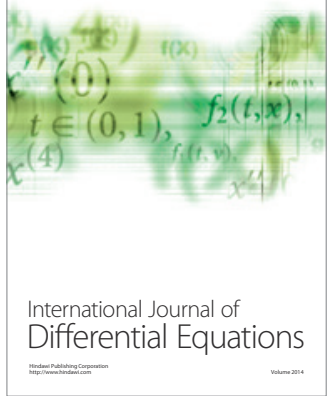
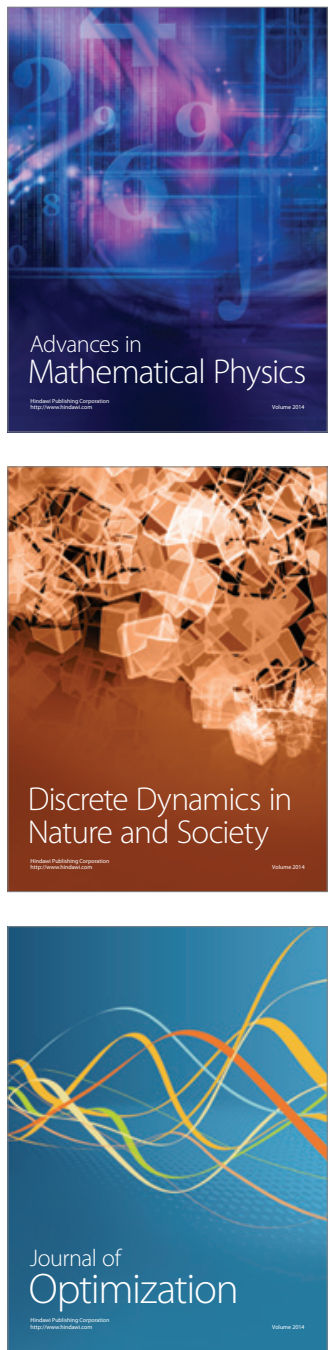